\title{
Neutrophil CD64 in diagnosis of infection in systemic lupus erythematosus patients
}

Enas E. El-Said ${ }^{1}$, Salwa R. Ali ${ }^{2}$ and Fawzia A. El-Sheshtawy ${ }^{3}$

${ }^{1,3}$ Clinical Pathology department; ${ }^{2}$ Internal medicine department, Faculty of medicine for girls, Al-Azhar university

\begin{abstract}
Background: Infection is one of the major complications as well as cause of death in systemic lupus erythematosus patients (SLE). Differentiation between early infection and disease flare in these patients is often clinically difficult because both have similar signs and symptoms.
\end{abstract}

Aim: To evaluate CD64 expression on neutrophils as an early marker that can discriminate between infection and disease flare in SLE patients. Also, its clinical utility in comparison with traditional laboratory tests used for detecting infection will be studied.

Methods: The study included 38 subjects; 10 apparently healthy individuals as healthy controls and 28 SLE patients divided into three groups (10 SLE patients with infection, 10 SLE patients with flare and 8 SLE patients without infection or flare). CD64 on neutrophils was measured using flow cytometry. Total leucocytic count, erythrocyte sedimentation rate and high-sensitive C-reactive protein were also measured.

Results: The median of the percentage of neutrophils expressing CD64 was higher in all SLE patients compared to normal control. It was significantly higher in SLE patients with infection than those with disease activity ( $\mathrm{P}<0.001$ ). Using a cutoff value of $\geq 17.6, \%$ of neutrophils expressing CD64; it revealed 100\% sensitivity and 100\% specificity.

Conclusion: The results of the present work showed that measurement of CD64 expression on neutrophils could be used as a sensitive and specific marker for detection of infection in SLE patients and differentiation between infection and disease activity.

Key words: Systemic lupus erythematosus, CD64, Infection.

\section{Introduction}

Infection is an important issue in systemic lupus erythematosus (SLE) patients because it is not only one of the major complications but also one of the most frequent causes of death among those patients (Cervera et al., 2003). This may be due to the immunomodulatory mechanisms occurring in SLE or immunosuppression caused by treatment with corticosteroids or cytotoxic drugs (Doran et al., 2002).

The risk for infection increases with age, presence of leucopenia, diabetes, chronic lung disease and prolonged corticosteroid treatment (Doran et al., 2002). Distinguishing active lupus from infection is often difficult because such patients may present initially with signs and symptoms of non specific inflammation such as fever, malaise, arthralgia and myalgia which could be due to either infection or disease flare (Reginato et al., 1994). However, such differentiation is important because the treatment differs dramatically (Matsui et al., 2006).

Traditionally, there is a battery of laboratory tests used for detecting infection including: total leucocytic count, presence of immature leucocyte forms, Creactive protein (CRP) and erythrocyte sedimentation rate (ESR). However, they can be misleadingly low in patients with systemic infections who are receiving corticosteroids or cytotoxic drugs. On the 
other hand, they can be misleadingly high in those with activity (Allen et al., 2002). Radiological findings or other diagnostic imaging lack sensitivity in the early stages of infection. Microbiological cultures are time consuming and may yield falsely negative results. Therefore, a highly specific and sensitive marker for infection that can provide rapid and easy result is extremely required (Matsui et al., 2006). Several molecules involved in the inflammatory response have been investigated as potential markers for infection. These include acute phase reactants (serum amyloid A and lipoplysccharaide binding protein), proand anti-inflammatory cytokines (IL6, IL8 and interferon gamma) and cellular surface markers (CD11, CD16, CD32 and CD64). Of the latter group, CD64 expression seems to have the highest diagnostic potential (Hoffmann, 2009).

Human defenses against bacteria are highly dependent on the cells of the innate immune system particularly polymorph nuclear leucoctytes (PNL). PNL recognize bacterial antigen through surface receptors including receptors for $\mathrm{FC}$ portion of immunoglobulin $\mathrm{G}$ or $\mathrm{FC} \gamma$ receptors $(\mathrm{FC} \gamma$ $\mathrm{R})$. There are three classes of IgG receptors: FC $\gamma$ RI, FC $\gamma$ RII and FC $\gamma$ RIII which can be recognized by the monoclonal antibodies CD64, CD32 and CD16 respectively (Zola et al., 2007). However, differences in expression between resting and activated neutrophils are much higher for FC $\gamma$ RI. Therefore, the CD64 antigen is the most useful marker for systemic infection and sepsis (Davis et al., 2006).

The aim of this study was to investigate the diagnostic value of CD64 expression on PNL as a marker that can distinguish early systemic infection from disease flare in SLE patients when signs and symptoms are non specific.

\section{Subjects and methods}

This study was carried out on 28 patients with systemic SLE admitted to the Internal Medicine Department of Al-zahraa Hospital Al- Azhar University between September 2009 and July 2010.They were diagnosed according to the revised criteria of American college of Rheumatology for SLE (Hochberg, 1997). Eight cases had inactive SLE, 10 cases had active SLE and 10 cases had SLE with infection. Consent was obtained from all patients before inclusion in the study. Also a case-control group of ten subjects was included in the study.

The patients and control were divided into the following groups.

1. Control group: consisted of 10 age matched apparently healthy individuals with a mean age \pm SD of $32 \pm 5.3$ years.

2. Inactive group: consisted of 8 SLE patients without evidence of disease activity with a mean age \pm SD of 29.4 \pm 7.5 years.

3. Active group: consisted of 10 SLE patients; they were diagnosed according systemic lupus erythematosus disease activity index (SLEDAI) for SLE (Bombardier et al., 1992) with a mean age \pm SD of $31.9 \pm 6.6$ years.

4. Infection group: consisted of 10 SLE patients with infections with a mean age \pm SD of $27 \pm 3.9$ years. Infection was diagnosed on clinical basis (e.g fever, cough, purulent sputum and chest crepitation for lower respiratory tract infection, dysuria, frequency and burning micturation for urinary tract infection). Blood samples and relevant microbiological samples were collected after the patients started empirical antibiotic treatment.

All studied participants were subjected to the following:

1- $\quad$ Full history and complete clinical examination. All patients were receiving varying doses of corticosteroids according to disease activity. Seven SLE active patients were also receiving cyclophosphamide for treatment of lupus nephritis. Six of the inactive SLE patients were receiving DMARD (Hydroxychloroquine).

2- Laboratory investigations:

Five $\mathrm{ml}$ of peripheral venous blood were withdrawn; under aseptic condition, from each patient and control .They were divided as follows: 
- $2.5 \mathrm{ml}$ anticoagulated with EDTA for complete blood picture, ESR and neutrophil CD64 flow cytometric analysis.

- $2.5 \mathrm{ml}$ without anticoagulant for high sensitive C-reactive protein (hsCRP), anti - ds DNA, C3 and C4 levels.

- Complete blood count was done by automated cell counter model Sysmex Kx N 21.

- Erythrocyte sedimentation rate was done by Westergren method.

- hs-CRP was done by ELISA using a complete set of ELISA reader model SLT Spectra 216687. The Kits was supplied by Monobind Inc. CA, USA. (Product code 3125-300).

- Detection of Anti - dsDNA autoantibody by ELISA using a complete set of ELISA reader model SLT Spectra 216687. The Kits was supplied by Orgentec diagnostika, Germany. (Product code 1435).

- Quantitative C3 and C4 were done by Radial immunodiffusion.

Preparation of surface CD64 for measurement by flow cytometer model (BECKMAN COULTER MACHINE):

CD 64 expression on neutrophils was measured by staining $100 \mathrm{ul}$ of whole blood with 10 ul anti - CD64 - FITC. All Kits supplied by (BD PharMingen Technical Data Sheet) (Catalog No 555527 Was: 31844X). Then incubated for 30 minutes at room temperature. 1.5 $\mathrm{ml}$ of lyse solution (NH4cl buffered with $\mathrm{KHCO} 3$ at $\mathrm{PH} 7.2$ ), were added to lyse RBCs. Then vortex and incubate for 10 minutes at room temperature in the dark. The tubes were centrifuged and supernatant was discarded, leaving $50 \mathrm{ul}$ of residual fluid in each tube to avoid disturbing the pellet, $0.5 \mathrm{ml}$ of phosphate buffered saline (PBS) was added to cell pellet then analysis by flow cytometry. Polymorphonuclear leukocytes were distinguished from other leukocyte by their characteristic side scatter. The gate was made on neutrophils stained with anti - CD64 against side scatter. The percentage of neutrophils expressing CD64 marker was identified.

\section{Statistical analysis}

The percentage of neutrophils expressing CD64 from the four groups of subjects was reported as median values. Normally distributed data (parametric data) were compared by analysis of variance (ANOVA) test to evaluate whether there was any evidence that the means of the different groups differ. If the ANOVA showed statistical difference, Tukey's multiple comparison test was used to compare between each two groups. Non parametric results were examined by the Kruskal -Wallis test and for the comparison between each two groups Mann - Whitney test was used. This was undertaken by a commercial software package (Epi-Info version 6) where $\mathrm{P}$ $<0.05$ was considered as significant. Spearman's correlation was used to assess the relation between CD64 and other laboratory parameters. The receiver operator criteria (ROC) analysis was used to obtain the optimum performance for CD64. 


\section{Results}

Table (1): Comparison between control group and inactive SLE group as regard studied parameters using Tukey's test.

\begin{tabular}{|c|c|c|c|c|}
\hline & $\begin{array}{c}\text { Control }(\mathbf{n}=10) \\
\text { mean } \pm \text { SD } \\
(\text { range }) \\
\end{array}$ & $\begin{array}{c}\text { Inactive }(\mathbf{n}=\mathbf{8}) \\
\text { mean } \pm \text { SD } \\
(\text { range })\end{array}$ & P-value & Significance \\
\hline Age (years) & $\begin{array}{c}32 \pm 5.3 \\
(24-40)\end{array}$ & $\begin{array}{c}29.4 \pm 7.5 \\
(22-45)\end{array}$ & 0.723 & NS \\
\hline $\mathrm{TLC} / \mu \mathrm{L}$ & $\begin{array}{l}6.4 \pm 1.15 \\
(4.6-8.1)\end{array}$ & $\begin{array}{c}7.2 \pm 2.3 \\
(4.4-10.0)\end{array}$ & 0.967 & NS \\
\hline $\operatorname{ESR}(\mathbf{m m} / \mathbf{h})$ & $\begin{array}{c}7.7 \pm 2.0 \\
5.0-11.0\end{array}$ & $\begin{array}{c}42.5 \pm 12.4 \\
(25.0-60.0)\end{array}$ & 0.095 & NS \\
\hline PLT/ $\mu \mathrm{L}$ & $\begin{array}{c}214.5 \pm 25.8 \\
(180.0-250.0)\end{array}$ & $\begin{array}{c}274.7 \pm 21.5 \\
240.0-300.0)\end{array}$ & 0.349 & NS \\
\hline $\mathrm{HB}(\mathrm{g} / \mathrm{dL})$ & $\begin{array}{c}11.97 \pm 0.92 \\
(10.9-14.0)\end{array}$ & $\begin{array}{l}10.15 \pm 0.5 \\
(9.5-11.0)\end{array}$ & $0.011^{*}$ & $S$ \\
\hline UR(mg/dL) & $\begin{array}{c}24.7 \pm 6.82 \\
(15.0-38.0)\end{array}$ & $\begin{array}{l}31.75 \pm 5.5 \\
(24.0-40)\end{array}$ & 0.990 & NS \\
\hline CR(mg/dL) & $\begin{array}{c}0.81 \pm 0.19 \\
(0.5-1.1) \\
\end{array}$ & $\begin{array}{c}0.89 \pm 0.22 \\
(0.5-1.2) \\
\end{array}$ & 1.000 & NS \\
\hline $\begin{array}{l}\text { Anti- } \\
\text { dsDNA(IU/mL) }\end{array}$ & $\begin{array}{c}4.48 \pm 3.26 \\
(1.2-12.0) \\
\end{array}$ & $\begin{array}{l}60.4 \pm 25.98 \\
(24.0-90.0) \\
\end{array}$ & $<0.001 *$ & $S$ \\
\hline $\mathrm{C3}(\mathrm{mg} / \mathrm{dL})$ & $\begin{array}{c}141.0 \pm 15.36 \\
(129-166)\end{array}$ & $\begin{array}{c}104.1 \pm 35.13 \\
(63.0-160.0)\end{array}$ & $0.007 *$ & $S$ \\
\hline $\mathrm{C4}(\mathrm{mg} / \mathrm{dL})$ & $\begin{array}{c}28.36 \pm 5.8 \\
(21.8-38.0) \\
\end{array}$ & $\begin{array}{c}26.21 \pm 6.1 \\
(20.0-35.0) \\
\end{array}$ & 0.868 & NS \\
\hline $\begin{array}{l}\text { Hs- CRP } \\
\mu \mathrm{g} / \mathrm{mL}\end{array}$ & $\begin{array}{c}2.7 \pm 1.6 \\
(1.1-6.3)\end{array}$ & $\begin{array}{c}18.9 \pm 7.3 \\
(3.5-26.7)\end{array}$ & $<0.001 *$ & $S$ \\
\hline
\end{tabular}

TLC; total leucocytic count, ESR; erythrocyte sedimentation rate, PLT; platelets, HB; hemoglobin, UR; urea, CR; creatinine, C3, 4; complement 3and 4, hs-CRP; high sensitive C reactive protein.

Inactive SLE patients had significantly higher Anti-dsDNA and hs-CRP values and significantly lower $\mathrm{C} 3$ and $\mathrm{HB}$ values than normal control. However, other parameters showed no significant difference between the two groups. 
Table (2): Comparison between control group and active SLE group as regard studied parameters using Tukey's test.

\begin{tabular}{|c|c|c|c|c|}
\hline & $\begin{array}{c}\text { Control }(\mathbf{n}=\mathbf{1 0}) \\
\text { mean } \pm \text { SD } \\
(\text { range })\end{array}$ & $\begin{array}{c}\text { Active }(\mathbf{n}=\mathbf{1 0}) \\
\text { mean } \pm \text { SD } \\
(\text { range })\end{array}$ & P-value & Significance \\
\hline Age (years) & $\begin{array}{c}32 \pm 5.3 \\
(24-40)\end{array}$ & $\begin{array}{l}31.9 \pm 6.6 \\
(24-45)\end{array}$ & 0.999 & NS \\
\hline $\mathrm{TLC} / \mu \mathrm{L}$ & $\begin{array}{l}6.4 \pm 1.15 \\
(4.6-8.1) \\
\end{array}$ & $\begin{array}{l}5.18 \pm 1.48 \\
(2.9-7.7) \\
\end{array}$ & 0.860 & NS \\
\hline $\operatorname{ESR}(\mathbf{m m} / \mathbf{h})$ & $\begin{array}{c}7.7 \pm 2.0 \\
5.0-11.0 \\
\end{array}$ & $\begin{array}{c}69.1 \pm 47.67 \\
(23.0-155.0) \\
\end{array}$ & $0.001 *$ & S \\
\hline PLT/ $/ \mu L$ & $\begin{array}{c}214.5 \pm 25.8 \\
(180.0-250.0)\end{array}$ & $\begin{array}{c}247.6 \pm 119.0 \\
(160.0-490.0)\end{array}$ & 0.775 & NS \\
\hline $\mathrm{HB}(\mathrm{g} / \mathrm{dL})$ & $\begin{array}{c}11.97 \pm 0.92 \\
(10.9-14.0) \\
\end{array}$ & $\begin{array}{c}8.54 \pm 1.3 \\
(6.4-10.2) \\
\end{array}$ & $<0.001 *$ & $S$ \\
\hline $\mathrm{UR}(\mathrm{mg} / \mathrm{dL})$ & $\begin{array}{c}24.7 \pm 6.82 \\
(15.0-38.0)\end{array}$ & $\begin{array}{l}128.4 \pm 87.1 \\
(39.0-266.0)\end{array}$ & $<0.001 *$ & $S$ \\
\hline CR(mg/dL) & $\begin{array}{c}0.81 \pm 0.19 \\
(0.5-1.1)\end{array}$ & $\begin{array}{r}2.09 \pm 0.95 \\
(1.0-3.4) \\
\end{array}$ & 0.596 & NS \\
\hline $\begin{array}{l}\text { Anti- } \\
\text { dsDNA(IU/mL) }\end{array}$ & $\begin{array}{c}4.48 \pm 3.26 \\
(1.2-12.0)\end{array}$ & $\begin{array}{l}79.46 \pm 17.98 \\
(50.0-105.0)\end{array}$ & $<0.001 *$ & S \\
\hline $\mathrm{C3}(\mathrm{mg} / \mathrm{dL})$ & $\begin{array}{c}141.0 \pm 15.36 \\
(129-166)\end{array}$ & $\begin{array}{c}71.72 \pm 25.1 \\
(30.0-100) \\
\end{array}$ & $<0.001 *$ & $S$ \\
\hline $\mathrm{C4}(\mathrm{mg} / \mathrm{dL})$ & $\begin{array}{c}28.36 \pm 5.8 \\
(21.8-38.0)\end{array}$ & $\begin{array}{c}23.5 \pm 4.7 \\
(17.8-33.0)\end{array}$ & 0.287 & NS \\
\hline Hs- CRP $\mu \mathrm{g} / \mathrm{mL}$ & $\begin{array}{c}2.7 \pm 1.6 \\
(1.1-6.3)\end{array}$ & $\begin{array}{c}15.8 \pm 3.4 \\
(11.0-20.8)\end{array}$ & $<0.001 *$ & $S$ \\
\hline
\end{tabular}

TLC; total leucocytic count, ESR; erythrocyte sedimentation rate, PLT; platelets, HB; hemoglobin, UR; urea, CR; creatinine, C3, 4; complement 3and 4, hs-CRP; high sensitive C reactive protein.

Active SLE patients had significantly higher urea, ESR, Anti-dsDNA and hs-CRP values and significantly lower $\mathrm{C} 3$ and $\mathrm{HB}$ values than normal control. However, there was no significant difference between the two groups as regard other laboratory parameters. 
Table (3): Comparison between control group and infection SLE group as regard studied parameters using Tukey's test.

\begin{tabular}{|c|c|c|c|c|}
\hline & $\begin{array}{c}\text { Control }(\mathbf{n}=\mathbf{1 0}) \\
\text { mean } \pm \text { SD } \\
(\text { range })\end{array}$ & $\begin{array}{c}\text { Infection }(\mathbf{n}=10) \\
\text { mean } \pm \text { SD } \\
(\text { range })\end{array}$ & P-value & Significance \\
\hline Age (years) & $\begin{array}{c}32 \pm 5.3 \\
(24-40)\end{array}$ & $\begin{array}{l}27 \pm 3.9 \\
(20-33) \\
\end{array}$ & 0.204 & NS \\
\hline TLC $/ \mu L$ & $\begin{array}{l}6.4 \pm 1.15 \\
(4.6-8.1)\end{array}$ & $\begin{array}{c}11.96 \pm 6.0 \\
(7.7-28)\end{array}$ & $0.006^{*}$ & S \\
\hline $\operatorname{ESR}(\mathbf{m m} / \mathbf{h})$ & $\begin{array}{c}7.7 \pm 2.0 \\
5.0-11.0\end{array}$ & $\begin{array}{c}79 \pm 35.4 \\
(20.0-130.0)\end{array}$ & $<0.001 *$ & S \\
\hline PLT/ $/ \mu L$ & $\begin{array}{c}214.5 \pm 25.8 \\
(180.0-250.0)\end{array}$ & $\begin{array}{c}187.0 \pm 85.3 \\
(118.0-417.0) \\
\end{array}$ & 0.858 & NS \\
\hline HB(g/dL) & $\begin{array}{c}11.97 \pm 0.92 \\
(10.9-14.0) \\
\end{array}$ & $\begin{array}{c}9.74 \pm 1.5 \\
(7.0-11.8) \\
\end{array}$ & $0.001 *$ & S \\
\hline $\mathrm{UR}(\mathrm{mg} / \mathrm{dL})$ & $\begin{array}{c}24.7 \pm 6.82 \\
(15.0-38.0)\end{array}$ & $\begin{array}{c}61.9 \pm 45.78 \\
(16.0-175.0)\end{array}$ & 0.375 & $S$ \\
\hline $\mathrm{CR}(\mathrm{mg} / \mathrm{dL})$ & $\begin{array}{c}0.81 \pm 0.19 \\
(0.5-1.1)\end{array}$ & $\begin{array}{c}1.16 \pm 0.74 \\
(0.5-3.1)\end{array}$ & 0.378 & NS \\
\hline $\begin{array}{l}\text { Anti- } \\
\text { dsDNA(IU/mL) }\end{array}$ & $\begin{array}{c}4.48 \pm 3.26 \\
(1.2-12.0)\end{array}$ & $\begin{array}{c}67.9 \pm 22.2 \\
(30.0-98.0)\end{array}$ & $<0.001 *$ & $S$ \\
\hline $\mathrm{C3}(\mathrm{mg} / \mathrm{dL})$ & $\begin{array}{c}141.0 \pm 15.36 \\
(129-166)\end{array}$ & $\begin{array}{c}88.7 \pm 5.8 \\
(80.0-100.0)\end{array}$ & $<0.001^{*}$ & $S$ \\
\hline C4(mg/dL) & $\begin{array}{c}28.36 \pm 5.8 \\
(21.8-38.0) \\
\end{array}$ & $\begin{array}{c}28.34 \pm 6.7 \\
(19.9-38.0) \\
\end{array}$ & 1.000 & NS \\
\hline Hs- CRP $\mu \mathrm{g} / \mathrm{mL}$ & $\begin{array}{c}2.7 \pm 1.6 \\
(1.1-6.3)\end{array}$ & $\begin{array}{c}19.5 \pm 6.9 \\
(10.0-32.0)\end{array}$ & $<0.001^{*}$ & S \\
\hline
\end{tabular}

TLC; total leucocytic count, ESR; erythrocyte sedimentation rate, PLT; platelets, HB; hemoglobin, UR; urea, CR; creatinine, C3, 4; complement 3and 4, hs-CRP; high sensitive C reactive protein.

SLE patients with infection had significantly higher TLC, ESR, urea, Anti-dsDNA and hsCRP values and significantly lower C3 and HB values than normal control. However, there was no significant difference between the two groups as regard other laboratory parameters. 
Table (4): Comparison between inactive SLE group and active SLE group as regard studied parameters using Tukey's test.

\begin{tabular}{|c|c|c|c|c|}
\hline & $\begin{array}{c}\text { Inactive }(\mathbf{n}=\mathbf{8}) \\
\text { mean } \pm \text { SD } \\
(\text { range })\end{array}$ & $\begin{array}{c}\text { Active }(\mathbf{n}=10) \\
\text { mean } \pm \text { SD } \\
(\text { range })\end{array}$ & P-value & Significance \\
\hline Age (years) & $\begin{array}{c}29.4 \pm 7.5 \\
(22-45)\end{array}$ & $\begin{array}{l}31.9 \pm 6.6 \\
(24-45)\end{array}$ & 0.816 & NS \\
\hline TLC $/ \mu \mathrm{L}$ & $\begin{array}{c}7.2 \pm 2.3 \\
(4.4-10.0)\end{array}$ & $\begin{array}{c}5.18 \pm 1.48 \\
(2.9-7.7)\end{array}$ & 0.635 & NS \\
\hline $\operatorname{ESR}(\mathbf{m m} / \mathbf{h})$ & $\begin{array}{c}42.5 \pm 12.4 \\
(25.0-60.0) \\
\end{array}$ & $\begin{array}{c}69.1 \pm 47.67 \\
(23.0-155.0)\end{array}$ & 0.293 & NS \\
\hline PLT/ $/ \mu L$ & $\begin{array}{c}274.7 \pm 21.5 \\
240.0-300.0)\end{array}$ & $\begin{array}{c}247.6 \pm 119.0 \\
(160.0-490.0)\end{array}$ & 0.881 & NS \\
\hline HB(g/dL) & $\begin{array}{l}10.15 \pm 0.5 \\
(9.5-11.0)\end{array}$ & $\begin{array}{c}8.54 \pm 1.3 \\
(6.4-10.2)\end{array}$ & $0.034 *$ & S \\
\hline $\mathrm{UR}(\mathrm{mg} / \mathrm{dL})$ & $\begin{array}{l}31.75 \pm 5.5 \\
(24.0-40)\end{array}$ & $\begin{array}{c}128.4 \pm 87.1 \\
(39.0-266.0)\end{array}$ & $0.002^{*}$ & $S$ \\
\hline CR(mg/dL) & $\begin{array}{c}0.89 \pm 0.22 \\
(0.5-1.2)\end{array}$ & $\begin{array}{l}2.09 \pm 0.95 \\
(1.0-3.4)\end{array}$ & 0.682 & NS \\
\hline $\begin{array}{l}\text { Anti- } \\
\text { dsDNA(IU/mL) }\end{array}$ & $\begin{array}{l}60.4 \pm 25.98 \\
(24.0-90.0)\end{array}$ & $\begin{array}{c}79.46 \pm 17.98 \\
(50.0-105.0)\end{array}$ & 0.184 & NS \\
\hline $\mathrm{C3}(\mathrm{mg} / \mathrm{dL})$ & $\begin{array}{c}104.1 \pm 35.13 \\
(63.0-160.0)\end{array}$ & $\begin{array}{c}71.72 \pm 25.1 \\
(30.0-100)\end{array}$ & $0.024 *$ & $S$ \\
\hline C4(mg/dL) & $\begin{array}{c}26.21 \pm 6.1 \\
(20.0-35.0)\end{array}$ & $\begin{array}{c}23.5 \pm 4.7 \\
(17.8-33.0)\end{array}$ & 0.773 & NS \\
\hline Hs- CRP $\mu \mathrm{g} / \mathrm{mL}$ & $\begin{array}{c}18.9 \pm 7.3 \\
(3.5-26.7)\end{array}$ & $\begin{array}{c}15.8 \pm 3.4 \\
(11.0-20.8)\end{array}$ & 0.614 & NS \\
\hline
\end{tabular}

TLC; total leucocytic count, ESR; erythrocyte sedimentation rate, PLT; platelets, HB; hemoglobin, UR; urea, CR; creatinine, C3, 4; complement 3and 4, hs-CRP; high sensitive C reactive protein.

Active SLE patients had significantly higher urea level and significantly lower C3 and HB values than inactive SLE patients. However, other parameters showed no significant difference between the two groups. 
Table (5): Comparison between inactive SLE group and SLE with infection group as regard studied parameters using Tukey's test.

\begin{tabular}{|c|c|c|c|c|}
\hline & $\begin{array}{c}\text { Inactive }(\mathbf{n}=\mathbf{8}) \\
\text { mean } \pm \text { SD } \\
(\text { range })\end{array}$ & $\begin{array}{c}\text { Infection }(\mathbf{n}=\mathbf{1 0}) \\
\text { mean } \pm \text { SD } \\
(\text { range }) \\
\end{array}$ & P-value & Significance \\
\hline Age (years) & $\begin{array}{c}29.4 \pm 7.5 \\
(22-45)\end{array}$ & $\begin{array}{l}27 \pm 3.9 \\
(20-33)\end{array}$ & 0.830 & NS \\
\hline TLC $/ \mu \mathrm{L}$ & $\begin{array}{c}7.2 \pm 2.3 \\
(4.4-10.0)\end{array}$ & $\begin{array}{c}11.96 \pm 6.0 \\
(7.7-28)\end{array}$ & $0.032 *$ & S \\
\hline $\operatorname{ESR}(\mathbf{m m} / \mathbf{h})$ & $\begin{array}{c}42.5 \pm 12.4 \\
(25.0-60.0)\end{array}$ & $\begin{array}{c}79 \pm 35.4 \\
(20.0-130.0)\end{array}$ & 0.074 & NS \\
\hline PLT/ $/ \mu \mathrm{L}$ & $\begin{array}{c}274.7 \pm 21.5 \\
240.0-300.0) \\
\end{array}$ & $\begin{array}{c}187.0 \pm 85.3 \\
(118.0-417.0)\end{array}$ & 0.091 & NS \\
\hline HB(g/dL) & $\begin{array}{l}10.15 \pm 0.5 \\
(9.5-11.0) \\
\end{array}$ & $\begin{array}{c}9.74 \pm 1.5 \\
(7.0-11.8) \\
\end{array}$ & 0.877 & NS \\
\hline $\mathrm{UR}(\mathrm{mg} / \mathrm{dL})$ & $\begin{array}{l}31.75 \pm 5.5 \\
(24.0-40)\end{array}$ & $\begin{array}{c}61.9 \pm 45.78 \\
(16.0-175.0)\end{array}$ & 0.607 & NS \\
\hline CR(mg/dL) & $\begin{array}{c}0.89 \pm 0.22 \\
(0.5-1.2)\end{array}$ & $\begin{array}{c}1.16 \pm 0.74 \\
(0.5-3.1)\end{array}$ & 0.473 & NS \\
\hline $\begin{array}{l}\text { Anti- } \\
\text { dsDNA(IU/mL) }\end{array}$ & $\begin{array}{l}60.4 \pm 25.98 \\
(24.0-90.0)\end{array}$ & $\begin{array}{c}67.9 \pm 22.2 \\
(30.0-98.0)\end{array}$ & 0.838 & NS \\
\hline $\mathrm{C3}(\mathrm{mg} / \mathrm{dL})$ & $\begin{array}{c}104.1 \pm 35.13 \\
(63.0-160.0)\end{array}$ & $\begin{array}{c}88.7 \pm 5.8 \\
(80.0-100.0)\end{array}$ & 0.465 & NS \\
\hline C4(mg/dL) & $\begin{array}{c}26.21 \pm 6.1 \\
(20.0-35.0)\end{array}$ & $\begin{array}{c}28.34 \pm 6.7 \\
(19.9-38.0)\end{array}$ & 0.871 & NS \\
\hline Hs- CRP $\mu \mathrm{g} / \mathrm{mL}$ & $\begin{array}{c}18.9 \pm 7.3 \\
(3.5-26.7)\end{array}$ & $\begin{array}{c}19.5 \pm 6.9 \\
(10.0-32.0)\end{array}$ & 0.996 & NS \\
\hline
\end{tabular}

TLC; total leucocytic count, ESR; erythrocyte sedimentation rate, PLT; platelets, HB; hemoglobin, UR; urea, CR; creatinine, C3, 4; complement 3and 4, hs-CRP; high sensitive C reactive protein.

There was significant increase in TLC in SLE patients with infection in comparison with inactive SLE, while other laboratory parameters revealed no difference. 
Table (6): Comparison between active SLE group and SLE with infection group as regard studied parameters using Tukey's test.

\begin{tabular}{|c|c|c|c|c|}
\hline & $\begin{array}{c}\text { Active }(\mathbf{n}=10) \\
\text { mean } \pm \text { SD } \\
\text { (range) }\end{array}$ & $\begin{array}{c}\text { Infection }(\mathbf{n}=\mathbf{1 0}) \\
\text { mean } \pm \text { SD } \\
(\text { range })\end{array}$ & P-value & Significance \\
\hline Age (years) & $\begin{array}{l}31.9 \pm 6.6 \\
(24-45) \\
\end{array}$ & $\begin{array}{l}27 \pm 3.9 \\
(20-33) \\
\end{array}$ & 0.288 & NS \\
\hline $\mathrm{TLC} / \boldsymbol{\mu L}$ & $\begin{array}{l}5.18 \pm 1.48 \\
(2.9-7.7) \\
\end{array}$ & $\begin{array}{c}11.96 \pm 6.0 \\
(7.7-28) \\
\end{array}$ & $0.001 *$ & S \\
\hline $\operatorname{ESR}(\mathbf{m m} / \mathbf{h})$ & $\begin{array}{c}69.1 \pm 47.67 \\
(23.0-155.0)\end{array}$ & $\begin{array}{c}79 \pm 35.4 \\
(20.0-130.0)\end{array}$ & 0.894 & NS \\
\hline PLT/ $/ \mu L$ & $\begin{array}{c}247.6 \pm 119.0 \\
(160.0-490.0)\end{array}$ & $\begin{array}{c}187.0 \pm 85.3 \\
(118.0-417.0)\end{array}$ & 0.327 & NS \\
\hline $\mathrm{HB}(\mathrm{g} / \mathrm{dL})$ & $\begin{array}{c}8.54 \pm 1.3 \\
(6.4-10.2)\end{array}$ & $\begin{array}{c}9.74 \pm 1.5 \\
(7.0-11.8) \\
\end{array}$ & 0.127 & NS \\
\hline $\mathrm{UR}(\mathrm{mg} / \mathrm{dL})$ & $\begin{array}{l}128.4 \pm 87.1 \\
(39.0-266.0)\end{array}$ & $\begin{array}{c}61.9 \pm 45.78 \\
(16.0-175.0) \\
\end{array}$ & $0.027 *$ & S \\
\hline $\mathrm{CR}(\mathrm{mg} / \mathrm{dL})$ & $\begin{array}{l}2.09 \pm 0.95 \\
(1.0-3.4) \\
\end{array}$ & $\begin{array}{c}1.16 \pm 0.74 \\
(0.5-3.1) \\
\end{array}$ & 0.988 & NS \\
\hline $\begin{array}{l}\text { Anti- } \\
\text { dsDNA(IU/mL) }\end{array}$ & $\begin{array}{l}79.46 \pm 17.98 \\
(50.0-105.0)\end{array}$ & $\begin{array}{l}67.9 \pm 22.2 \\
(30.0-98.0)\end{array}$ & 0.552 & NS \\
\hline C3 (mg/dL) & $\begin{array}{c}71.72 \pm 25.1 \\
(30.0-100) \\
\end{array}$ & $\begin{array}{c}88.7 \pm 5.8 \\
(80.0-100.0) \\
\end{array}$ & 0.353 & NS \\
\hline C4(mg/dL) & $\begin{array}{c}23.5 \pm 4.7 \\
(17.8-33.0)\end{array}$ & $\begin{array}{c}28.34 \pm 6.7 \\
(19.9-38.0)\end{array}$ & 0.291 & NS \\
\hline $\begin{array}{l}\text { Hs- CRP } \\
\mu \mathrm{g} / \mathrm{mL}\end{array}$ & $\begin{array}{c}15.8 \pm 3.4 \\
(11.0-20.8) \\
\end{array}$ & $\begin{array}{c}19.5 \pm 6.9 \\
(10.0-32.0)\end{array}$ & 0.427 & NS \\
\hline
\end{tabular}

TLC; total leucocytic count, ESR; erythrocyte sedimentation rate, PLT; platelets, HB; hemoglobin, UR; urea, CR; creatinine, C3, 4; complement 3and 4, hs-CRP; high sensitive C reactive protein.

Active SLE patients had significantly higher urea level and significantly lower TLC than SLE patients with infection. However, other parameters showed no significant difference between the two groups.

The infection group was diagnosed and started antibiotic treatment on clinical and radiological basis without waiting for culture results. Seven patients had chest infection; only 3 showed positive sputum culture, 3 patients with urinary tract infection; only 2 had positive urine culture (Table: 7).

Table (7): The culture results of the infection group.

\begin{tabular}{lccc}
\hline Infection type & Culture & \multicolumn{2}{c}{ Culture Positive } \\
\cline { 3 - 4 } & Negative & Gram +ve & Gram -ve \\
\hline Chest infection $(\mathrm{n}=7)$ & 4 & 1 & 2 \\
\hline UTI $(\mathrm{n}=3)$ & 1 & - & 2 \\
\hline
\end{tabular}




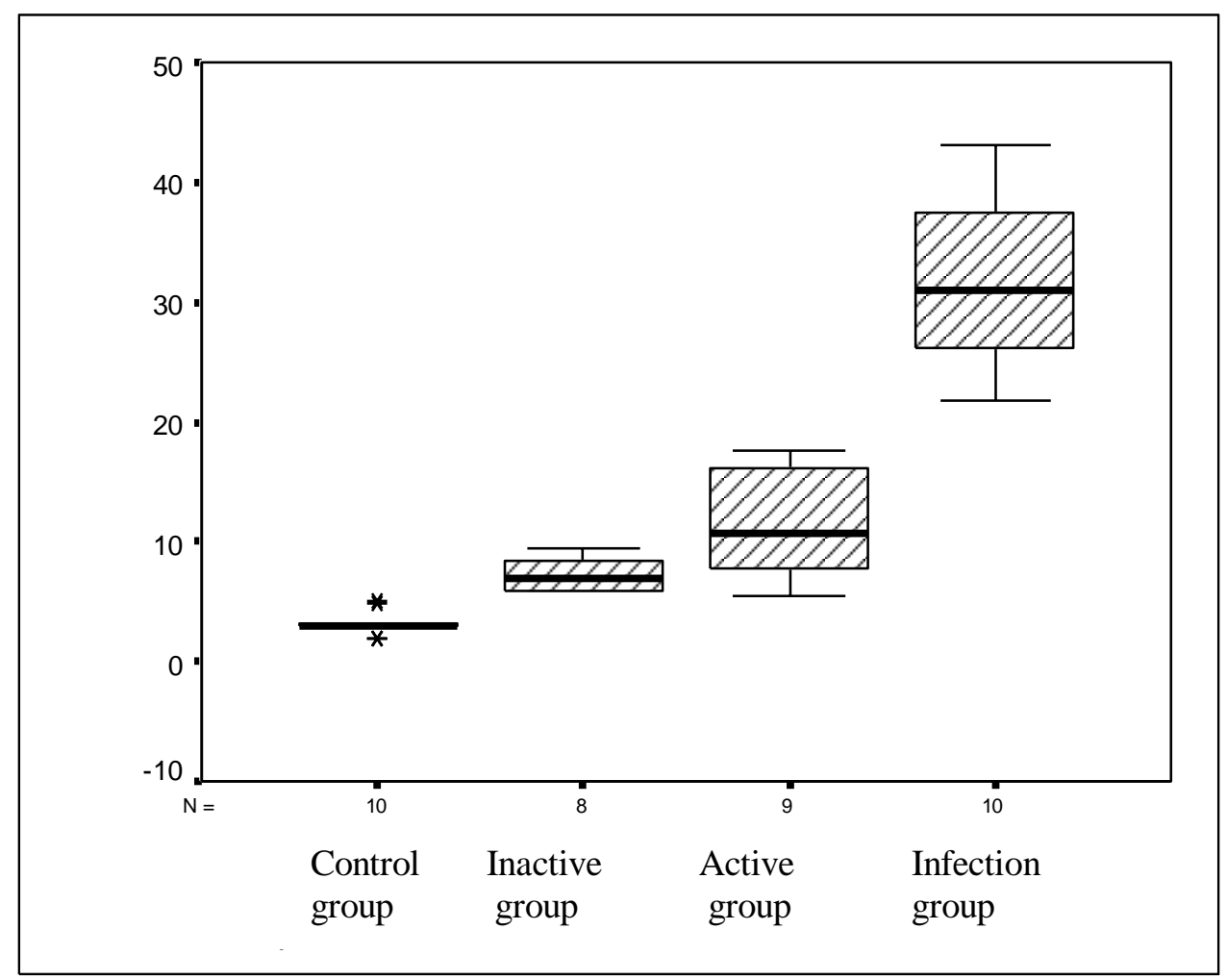

Figure (1): A box plot of the \% of neutrophils expressing CD64 for the normal control and the three patient groups.

Table (8): Comparison between the median of \% of neutrophil expressing CD64 in the control group and all patient groups using Mann-Whitney test.

\begin{tabular}{cccc}
\hline $\begin{array}{c}\text { Group } \\
\text { Median (range) }\end{array}$ & $\begin{array}{c}\text { Control group } \\
2.96(1.86-5.1)\end{array}$ & Inactive SLE group & Active SLE group \\
\hline Inactive $(\mathbf{n}=\mathbf{8})$ & $\mathrm{P}<0.001^{*}$ & - & $\mathrm{P}=0.093$ \\
$6.85(5.84-9.36)$ & & $\mathrm{P}=0.093$ & - \\
$\begin{array}{c}\text { Active }(\mathbf{n}=\mathbf{1 0}) \\
10.6(5.4-17.6)\end{array}$ & $\mathrm{P}<0.001^{*}$ & & $\mathrm{P}<0.001^{*}$ \\
Infection $(\mathbf{n = 1 0})$ & $\mathrm{P}<0.001^{*}$ & $\mathrm{P}<0.001^{*}$ \\
$31.0(21.7-43.0)$ & &
\end{tabular}

The percentage of neutrophil expressing CD64 was significantly higher in all SLE compared to the control group $(\mathrm{P}<0.001)$. There was no statistical difference between inactive and active groups $(\mathrm{P}=0.093)$. In patients with infection, \% of neutrophil expressing CD64 was significantly higher than the active group $(\mathrm{P}<0.001)$. 
The figures 2-5 show selected histograms for percentage of neutrophil expressing CD64 in some studied patients.

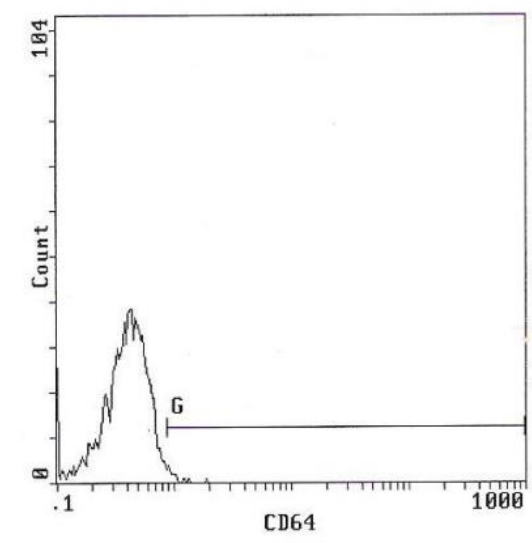

Figure (2): a histogram of a control subject showing neutrophil expressing CD64 $=1.86$ $\%$

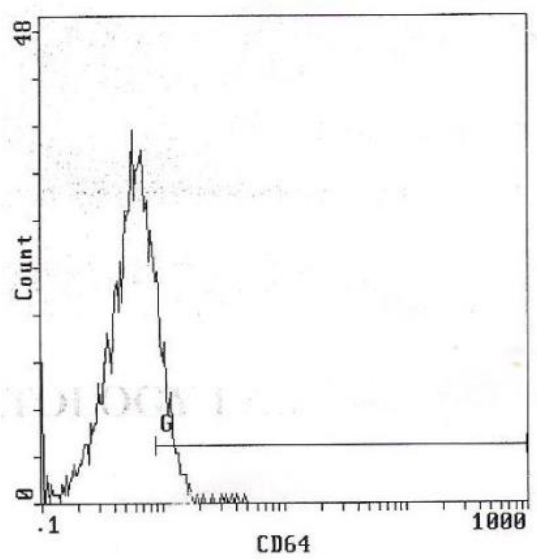

Figure (4): a histogram of a case of active SLE showing neutrophil expressing CD64 = $17.6 \%$

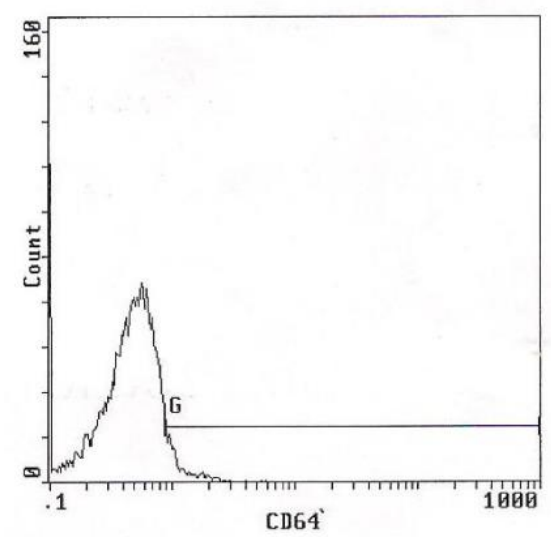

Figure (3): a histogram of a case of inactive SLE showing neutrophil expressing CD64 = $7.67 \%$

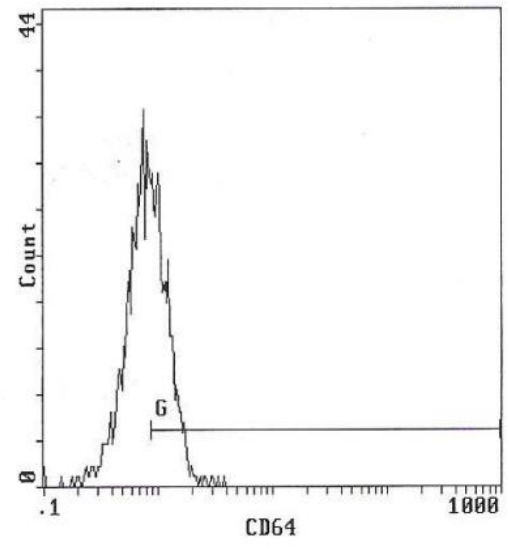

Figure (5): a histogram of a case of SLE with infection showing neutrophil expressing CD64 $=43.0 \%$ 
Using the results of the \% of neutrophil expressing CD64 in the active group and infection group with receiver operating criteria (ROC) analysis, a cutoff value of $17.6 \%$ was found to be sensitive and specific for differentiating infection from flare in SLE patients. (Table: 9) (fig: 6).

Table (9): Cutoff level, sensitivity, specificity, positive predictive value and negative predictive value for percentage of neutrophils expressing CD64.

\begin{tabular}{cccccc} 
Cutoff & Sens. & Spec. & PPV & NPV & Accuracy \\
\hline$>17.6 \%$ & 100.0 & 100.0 & 100.0 & 100.0 & 100.0
\end{tabular}

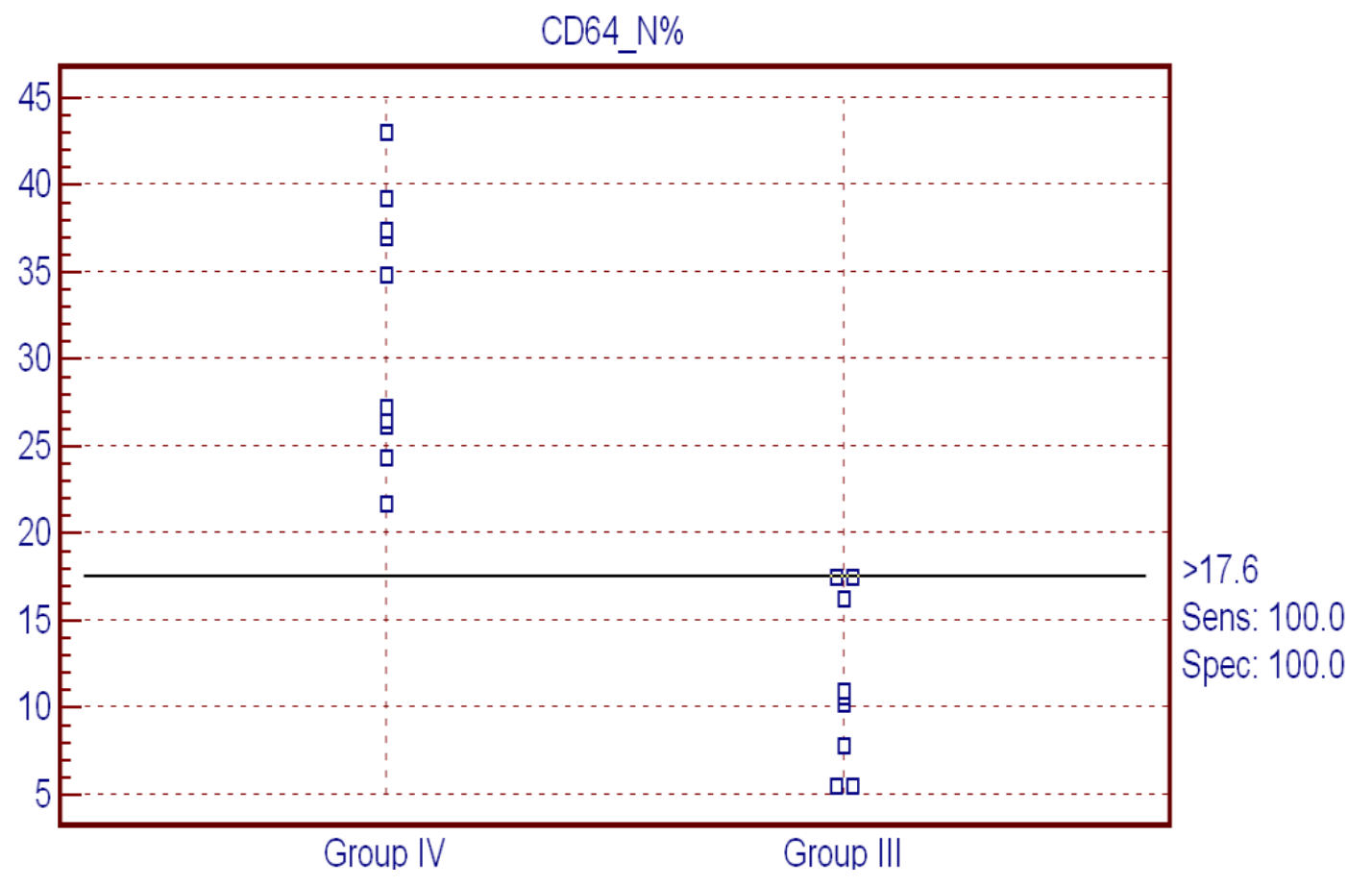

Figure (6): ROC curve for percentage of CD64 expressing neutrophils 
Correlation between \% of neutrophil expressing CD64 and other laboratory parameters

Table (10): Correlation between \% of neutrophil expressing CD64 and other laboratory parameters.

\begin{tabular}{|c|c|c|c|}
\hline & & \multicolumn{2}{|c|}{ CD64 \% } \\
\hline & & $\mathbf{R}$ & P-value \\
\hline \multirow{4}{*}{$\mathrm{TLC} / \mu \mathrm{L}$} & Control group & -0.363 & 0.302 \\
\hline & Inactive group & 0.104 & 0.806 \\
\hline & Active group & -0.653 & 0.057 \\
\hline & Infection group & 0.157 & 0.666 \\
\hline \multirow{4}{*}{$\operatorname{ESR}(\mathbf{m m} / \mathbf{h})$} & Control group & 0.416 & 0.231 \\
\hline & Inactive group & 0.266 & 0.524 \\
\hline & Active group & -0.341 & 0.369 \\
\hline & Infection group & -0.180 & 0.619 \\
\hline \multirow{4}{*}{$\begin{array}{c}\text { Anti- } \\
\text { dsDNA(IU/mL) }\end{array}$} & Control group & -0.012 & 0.974 \\
\hline & Inactive group & -0.446 & 0.268 \\
\hline & Active group & 0.622 & 0.074 \\
\hline & Infection group & 0.284 & 0.426 \\
\hline \multirow{4}{*}{$\mathrm{C} 3$ (mg/dL) } & Control group & 0.053 & 0.885 \\
\hline & Inactive group & 0.031 & 0.942 \\
\hline & Active group & 0.247 & 0.522 \\
\hline & Infection group & -0.337 & 0.341 \\
\hline \multirow{4}{*}{$\mathrm{C} 4(\mathrm{mg} / \mathrm{dL})$} & Control group & -0.060 & 0.869 \\
\hline & Inactive group & 0.511 & 0.195 \\
\hline & Active group & -0.120 & 0.759 \\
\hline & Infection group & 0.177 & 0.625 \\
\hline \multirow{4}{*}{ Hs- CRP $\mu \mathrm{g} / \mathrm{mL}$} & Control group & -0.259 & 0.469 \\
\hline & Inactive group & -0.163 & 0.700 \\
\hline & Active group & -0.105 & 0.788 \\
\hline & Infection group & -0.085 & 0.815 \\
\hline
\end{tabular}

TLC; total leucocytic count, ESR; erythrocyte sedimentation rate, PLT; platelets, HB; hemoglobin, UR; urea, CR; creatinine, C3, 4; complement 3and 4, hs-CRP; high sensitive C reactive protein.

There was no correlation between \% of neutrophil expressing CD64 and other laboratory parameters including those used for diagnosis of infection in SLE patients.

\section{Descriptive data:}

A 23 years old female patient was presented with convulsions and diagnosed as lupus cerebri (SLE flare). She was on steroids and cyclophosphamide for the last 9 months for lupus nephritis. She had no clinical or laboratory evidence of infection. She received pulse steroid therapy and prophylactic antibiotic treatment and discharged. She had $36.2 \%$ of neutrophils expressing CD64 (above the cutoff level to diagnose infection) One week later, she presented by fever, cough, purulent sputum, bronchopneumonia (by X-ray) and died. Her laboratory investigations are shown in table (11). 
Table (11): Labortatory data of the lupus cerebri patient at initial presentation and one week later.

\begin{tabular}{ccc}
\hline & Initial presentation & One week later \\
\hline $\mathbf{T L C} / \boldsymbol{\mu L}$ & 10.5 & 28 \\
$\mathbf{E S R}(\mathbf{m m} / \mathbf{h})$ & 34 & 55 \\
Hs- CRP $\boldsymbol{\mu \mathbf { g } / \mathbf { m L }}$ & 24.4 & 32 \\
\% of Neutrophil & 36.2 & 39.2 \\
expressing CD64 & 180 & 195 \\
$\mathbf{U R}(\mathbf{m g} / \mathbf{d L})$ & 3.0 & 3.2 \\
CR $(\mathbf{m g} / \mathbf{d L})$ & &
\end{tabular}

These data could reveal the presence of hidden infection which might be evidenced by the elevated percentage of neutrophils expressing CD64 at initial presentation.

\section{Discussion}

Systemic lupus erythematosus is a chronic relapsing disorder of connective tissue, characterized principally by involvement of the skin, joints, kidneys and serosal membranes. It is of unknown etiology, but is thought to represent a failure of the regulatory mechanisms of the autoimmune system (Edworthy, 2001).

Infection is responsible for approximately $25 \%$ of deaths in patients with SLE, making it a leading cause of mortality among SLE patients (Goldblatt et al., 2009).

The reasons behind the high rate of infection seen among patients with SLE are multifactorial. Impaired immune system defenses can be caused by active disease, SLE-associated dysfunction of both innate and acquired immunity, or by the immunosuppressive medications used to treat SLE. Additionally, patients have greater exposure to infectious pathogens, particularly drug resistant ones, from proximity to other patients and health care workers during outpatient and/or inpatient visits (Kamen, 2009).

Infection in SLE patients is often hard to detect clinically because of non specific symptoms and signs that do not enable physicians to differentiate between infection and flare of the underlying disease (Matsui et al., 2006). Patients are often empirically treated for both possibilities with broad spectrum antibiotics and high dose steroids. The complexity of the clinical presentations of both infection and flare requires investigation of new markers that may be of diagnostic value rather than the poor traditional markers (Allen et al., 2002).

The total leucocytic count is not a reliable marker of infection in SLE patients because of many factors including leucopenia in SLE activity, immunosuppressive treatment and myelotoxicity caused by cytotoxic drugs. On the other hand, in many cases of sepsis TLC is increased although it can also be normal or even decreased. In addition, the TLC gives no information about the functional status of leukocytes (Hoffmann, 2009).

For a long time CRP has been regarded the best diagnostic test available for infection and sepsis. However, most SLE patients without infection frequently display clinically significant CRP levels

(Gaitonde et al., 2008).

CRP is significantly increased in SLE patients suffering from intercurrent infection (de Carvalho et al., 2007). CRP levels above $60 \mathrm{mg} / \mathrm{l}$ in febrile SLE patients without serositis almost always indicate infection. However, such high level of CRP is only reached after the infection become severe (ter Borg et al., 1990 and Roy and Tan 2001). This is because; SLE patients have a significantly lower CRP response that may be due to the underlying immunoregulatory mechanisms such as consumption by immune complexes, genetic factors, as well as concomitant use of corticosteroids. Whereas in SLE alone, CRP levels are only moderately raised even in patients with very active disease (Nikiphorou 
and Pyne, 2009).

The erythrocyte sedimentation rate is frequently elevated during the course of active SLE, and in lupus in remission may remain elevated for long peroids of time (Sox and Liang, 1986).

Neutrophil CD64 has many characteristes that make it clinically useful as a marker of sepsis. First, CD64 directly reflects physiologic events of the inflammation response to invading microorganisms and CD64 is functionally correlated with phagocytosis. Secondly, in resting neutrophils the level of CD64 expression is rather low. However following activation it can increase up to $5-10-$ folds, allowing good discrimination between health and disease. Third, neutrophil CD64 becomes positive rapidly, upregulation of CD64 is demonstrable within $4-6$ hs following contact of neutrophils with proinflammatory cytokines granulocyte colony stimulating factor ( $\mathrm{G}-\mathrm{CSF}$ ) ind interferon gamma (IFN $-\gamma$ ) (Hoffmann, 2009).

The utility of CD64 to detect infection has been reported in several studies. In 1995, Davis and colleagues were the first to propose the diagnostic potential of neutrophil CD64 as a marker of infection (Davis et al., 1995). Muller Kobold et al., (2000) demonstrated a significant increase in neutrophil CD64 expression, with a median 10 - fold increase when compared with healthy controls for 17 patients with culture - proven sepsis.

The present study was carried out to identify the utility of measuring CD64 to differentiate between systemic infection and disease activity in patients with SLE. The median of the percentage of neutrophil expressing CD64 was significantly higher in the infection group $(31.0 \%)$, and in the active group $(10.6 \%)$ than in the inactive group $(6.85 \%)$ and control group $(1.86 \%)(\mathrm{P}<0.001)$. These results were similar to the study conducted by Allen et al., (2002). They reported for the first time statistical measures for the performance of CD64 as a diagnostic test to differentiate between systemic infection and active autoimmune inflammation. They found that CD64 was slightly increased in the autoimmune group, but much stonger in the group with systemic infection.

Matsui et al., (2006) reported that CD64 expression on neutrophils can be used to differentiate infection from an RA flare in a study included patients with rheumatoid arthritis (RA) in various stages of disease activity.

The present study showed increased CD64 expression on neutrophils in inactive SLE patients than in the control group and this may reflect the state of neutrophil activation by inflammatory state. In the absence of infection, non infectious stimuli such as trauma or inflammatory insult could be responsible for increased neutrophil CD64 (Qureshi et al., 2001). On the other hand, Szucs et al., (1995) have reported that CD64 expression of peripheral blood neutrophils in patients with SLE was not upregulated; however, the activity of SLE at the time of measurement was not addressed.

This study showed no significant correlations between \% of neutrophils expressing CD64 and markers of infection including total TLC, ESR and hs-CRP. These results were similar to the results of Muller Kobold et al (2000). They found no significant correlation between CD64 expression on neutrophils and traditional markers of infection (TLC, neutrophil count and CRP). Also, Allen et al (2002) found no correlation between CD64 expression and ESR.

As the microbiological samples in the present work were collected after starting antibiotic treatment, the number of culture positive samples was few which might reflect untrue results. Allen et al., (2002) reported that infections by Gram negative bacteria had higher CD64 expression on neutrophils compared with Gram positive infections due to different bacterial products activating neutrophils through different pathways. However, other reports did not support a significant difference between the type of bacteria (Matsui et al., 2006). On the other hand, it would be of interest to investigate whether daily monitoring of CD64 expression during antibiotic therapy could provide useful information with respect to discontinuation of drugs (Hoffmann, 
2009).

In this study, when a cutoff value $\geq 17.6 \%$ of neutrophils expressing CD64 used, it yielded $100 \%$ sensitivity and $100 \%$ specificity. So, it could be cosidered as a highly sensitive and specific marker to differentiate infection from disease activity in SLE patients. Matsui et al., (2006) reported sensitivity of $93 \%$ and specificity of 97\% for the CD64 for the diagnosis of infection and to discriminate it from RA activity. While Allen et al., (2002) reported sensitivity of $85 \%$ and specificity of $91 \%$.

In the present study, the SLE patients were on varying doses of immunosuppressive drug. Matsui et al., (2006) reported that CD64 expression was not affected by treatment with corticosteroids, DMARDS, or biological agents.

Analysis of descriptive data

The data obtained from this patient indicated that at the initial presentation there was no clinical evidence of infection at that time, the hs-CRP was only moderately elevated and the TLC was within normal while the $\%$ of neutrophils expressing CD64 was elevated above the cutoff value established in this study $(17.6 \%)$. One week later, the patients presented with bronchopneumnia that required ventilator, elevated TLC and hsCRP was still moderately elevated. This may be indicative that CD64 could be used as early and specific marker of sepsis in SLE patients.

\section{Conclusions}

Neutrophil expression of CD64 is a highly sensitive and specific marker for systemic infection and discriminating infection from disease flare up in patients with SLE. It has superior diagnostic value compared with the routine laboratory tests.

Measurement of CD64 expression on neutrophils can be performed easily and rapidly, so it is recommended to be used routinely in detection of infection. Further studies may be required to evaluate CD64 expression before and after antibiotic treatment.

\section{References}

Allen E, Bake AC, Purtzer Mz and Deodhar A (2002): Neutrophil CD64 expression: distinguishing acute inflammatory autoimmune disease from systemic infections. Ann Rheum Dis; 61: 522.

Bombardier C, Gladman DD, Urowitz MB, Caron D and Chang CH (1992): Derivation of the SLEDAI. A disease activity index for lupus patients. The committee on prognosis studies in SLE. Arthritis Rheum; 35 (6): 630.

Cervera R, Khamashta MA, Font J, Sebastiani GD, Gil A, Lavilla P, Mejía JC, Aydintug AO, Chwalinska-Sadowska H, de Ramón E, Fernández-Nebro A, Galeazzi M, Valen M, Mathieu A, Houssiau F, Caro N, Alba P, Ramos-Casals $M$, Ingelmo $M$ and Hughes GR (2003): European Working Party on Systemic Lupus Erythematosus.. Morbidity and mortality in systemic lupus erythematosus during a 10-year period: a comparison of early and late manifestations in a cohort of 1,000 patients. Medicine (Baltimore). 82: 299.

Davis BH, Biglow NC, Curnutte JT and Ornvold K (1995): Neutrophil CD64 expression: potential diagnostic indicator of acute inflammation and therapeutic monitor of interferon - gamma therapy. Lab Hematol; 1: 3.

Davis BH, Olsen SH, Ahmad E and Bigelow NC (2006): Neutrophil CD64 is an improved indicator of infection or sepsis in emergency department patients. Arch Pathol Lab Med; 130: 654.

de Carvalho JF, Hanaoka B, SzyperKravitz $M$ and Shoenfeld Y. Acta Reumatol Port (2007): C - Reactive protein and its implication in systemic lupus erythematosis. Acta Reumatol Port ; 32 (4): 317.

Doran MF, Crowson CS, Pond GR, O'Fallon WM and Gabriel SE (2002): Predictors of infection in rheumatoid arthritis. Arthritis Rheum; 46: 2294.

Edworthy SM (2001): Clinical manifestation of systemic lupus erythematosus. In Kelly's text book of rheumatology: 1105 .

Gaitonde S, Samols D, And Kushner I (2008): C-reactive protein and systemic lupus erythematosus. Arthritis \& Rheumatism (Arthritis Care \& Research)

59 (12): 1814.

Goldblatt F, Chambers S, Rahman $A$ and Isenberg DA (2009): Serious infections in British patients with systemic lupus erythematosus: hospitalisations and mortality. Lupus. 18: 682.

Hochberg MC (1997): Updating the American College of Rheumatology revised criteria for the classification of systemic lupus 
erythematosus Arthritis Rheum; 40: 1725.

Hoffmann JJ (2009): Neutrophil CD64: a diagnostic marker for infection and sepsis; Clin Chem Lab Med; 47(8): 903.

Kamen D (2009): How can we reduce the risk of serious infection for patients with systemic lupus erythematosus? Arthritis Res Ther; 5: 129

Matsui T, Ohsumi K, Ozawa N, Shimada K, Sumitomo S, Shimane K, Kawakami M, Nakayama H, Sugii S, Ozawa Y and Tohma S (2006): CD64 on neutrophils is a sensitive and specific marker for detection of infection in patients with rheumatoid arthritis. J Rheumatol; 33: 2416.

Muller Kobold AC, Tulleken JE, Zijlstra JG, Sluiter W, Hermans J, Kallenberg CG and Tervaert JW (2000): Leukocyte activation in sepsis; correlations with disease state and mortality. Intens Care Med; 26: 883.

Nikiphorou E and Pyne D (2009): Interpretation of $\mathrm{C}$ - reactive protein values in systemic lupus erythematosus; Grand Rounds; 9: 18.

Qureshi SS, Lewis SM, Gant VA, Treacher D, Davis BH and Brown KA (2001): Increased distribution and expression of CD64 on blood mpolymorphnuclear cells from patients with the systemic inflammatory response syndrome (SIRS). Clin EXP Immunol; 125: 258.

Reginato AJ and Falasca GF (1994): Fever in patients with rheumatic diseases. In:
Mandell BF, ed. Acute rheumatic and immunological diseases, management of the critically ill patient. New York: Marcel Decker; 125.

Roy S and Tan KT (2001): Pyrexia and normal C-reactive protein (CRP) in patients with systemic lupus erythematosus: always consider the possibility of infection in febrile patients with systemic lupus erythematosus regardless of CRP levels. Rheumatology, 40: 349.

Sox AS and Liang MH (1986): Measurements of systemic lupus activity in clinical researh. Arthritis Rheum; 31: 817.

Szucs G, Kavai M, Kiss E, Csipo I and Szegedi G (1995): Correlation of $\mathrm{IgG}$ Fc receptors on granulocytes with serum immune complex level in systemic lupus erythematosus. Scand J Immunol; 42: 577.

ter Borg EJ, Horst G, Limburg PC, van Rijswijk MH and Kallenberg CG (1990): C-reactive protein levels during disease exacerbations and infections in systemic lupus erythematosus: erythematosus: a prospective longitudinal study. J Rheumatol; 17: 1642.

Zola H, Swart B, Banham A, Barry S, Beare A, Bensussan A, Boumsell L, D Buckley C, Bühring HJ, Clark G, Engel P, Fox D, Jin BQ, Macardle PJ, Malavasi F, Mason D, Stockinger $H$, and Yang $X$ (2006): CD molecules 2006 - human cell differentiation molecules. J Immunol Meth; 319: 1. 


\section{س دـ 64 على الخلايا المتعادلة فى تثخيص العدوى فى مرضى الأنبة الحمراء}

ايناس السباعى السيد 1, سلوى رشاد على 2, و فوزية عبد السميع الثشتاوى 3

1 ، 3 قسم الباثولوجبا الاكلينكية و الكيميائية. 2 قسم الامر اض الباطنية ـ كلية الطب للبنات ، جامعة الأزهر

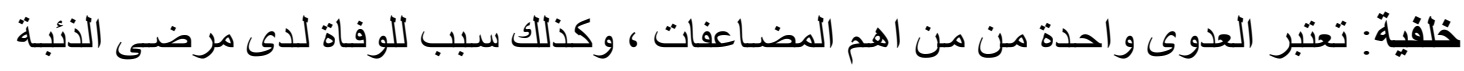

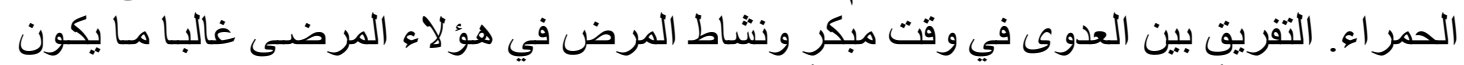
صعبا سريريا لأن كلا منهم لديه علاءمات و أعر اض مداثلة.

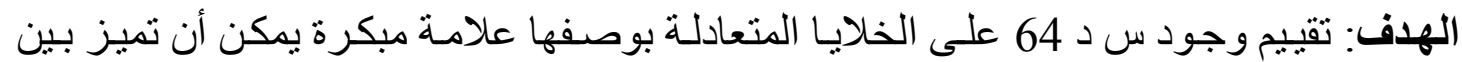

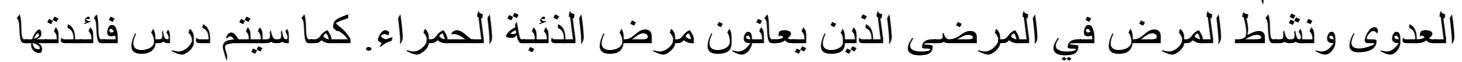

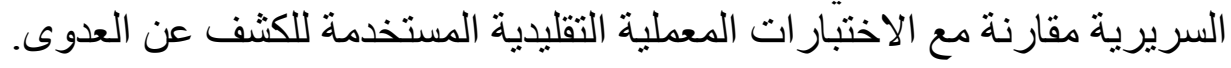

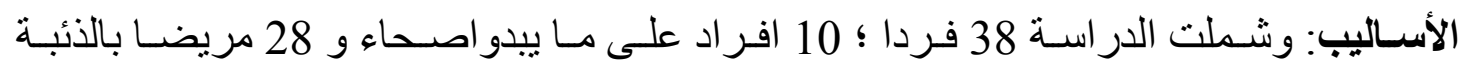

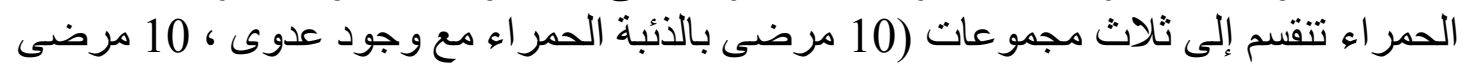

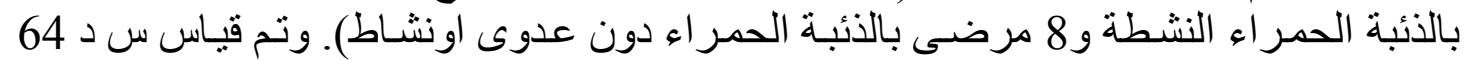
على الخلايا المتعادلة باستخدام التدفق الخلوي. سرعة الترسيب و البروتين التفاعلي سي عالى الحساسية.

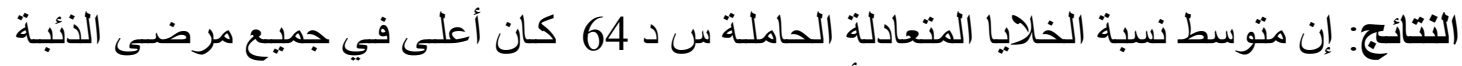

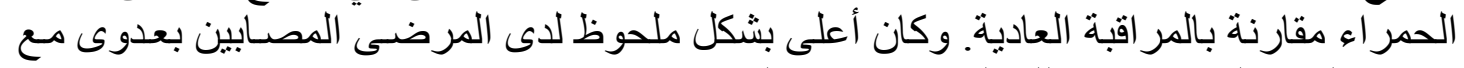

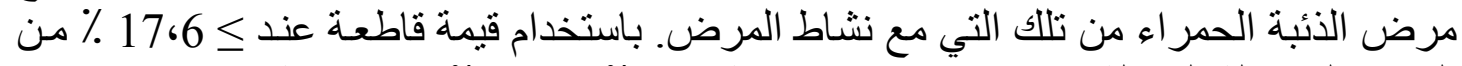
الخلايا المتعادلة الحاملة س د 64 ، تبين حساسية 100 \% و 100 \% خصوصية

الخلاصة: أظهرت نتائج هذه الدر اسـة أن قياس وجود س د 64 على الخلايـا المتعادلة يمكن أن

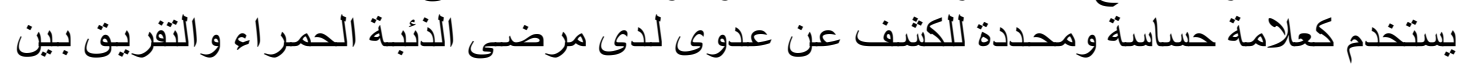

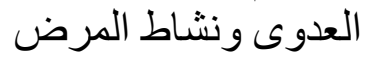
الكلمات الدالة : الذئبة الحمر اء، س د 64 ، و العدوى. 